\title{
Formen des Technologietransfers : Folgerungen für die Schweizer Entwicklungspolitik
}

Rolf Weder

\section{(2) OpenEdition \\ 1 Journals}

Electronic version

URL: http://journals.openedition.org/sjep/1220

DOI: $10.4000 /$ sjep. 1220

ISSN: 1663-9677

Publisher

Institut de hautes études internationales et du développement

Printed version

Date of publication: 1 janvier 1991

Number of pages: $235-253$

ISSN: 1660-5926

\section{Electronic reference}

Rolf Weder, «Formen des Technologietransfers : Folgerungen für die Schweizer Entwicklungspolitik », Schweizerisches Jahrbuch für Entwicklungspolitik [Online], 10 | 1991, Online erschienen am: 13 April 2013, abgerufen am 08 September 2020. URL : http://journals.openedition.org/sjep/1220 ; DOI : https://doi.org/10.4000/sjep.1220 


\title{
Formen des Technologietransfers: Folgerungen für die Schweizer Entwicklungspolitik
}

\author{
Rolf Weder
}

\section{Einleltung}

Die Schweiz gehört zu den kleinen Volkswirtschaften, die durch eine hohe und vielfältige internationale Verflechtung der Wirtschaft zu einem bedeutenden Wohlstand gekommen sind. Das im internationalen Vergleich einzigartige Ausmass der Verflechtung vieler Branchen mit dem Ausland gäbe ihr aber auch eine Chance, vermehrt zu einem im Ausland entwicklungsfördemden Knowhow-Transfer beizutragen. Dabei geht es aber nicht darum, irgendeine momentan gerade modische Technologie kostenlos oder mit Bundeskrediten in ein Entwicklungsland zu transferieren - sei dies nun eine 'angepasste' Handpumpe oder ein 'hochentwickelter' Reaktor. Vielmehr muss es das Ziel sein, weg von der 'Technologietransfer-Gläubigkeit' zu kommen, hin zu einer nüchternen Beurteilung und Förderung von bestimmten internationalen Transaktionsformen sowie der Bereitstellung des öffentlichen Gutes 'Information'. Gerade internationale Kooperationsformen stellen diesbezüglich für die öffentliche Entwicklungszusammenarbeit eine grosse Herausforderung dar. Damit die verschiedenen Transaktionsformen auch realisiert werden und ihre Wirkungen zeigen können, sind aber auch institutionelle Reformen - d.h. Veränderungen im politisch-ökonomischen System - in den Entwicklungsländern notwendig.

Im ersten Abschnitt folgt eine Definition und Differenzierung von verschiedenen Formen des Know-how-Transfers. Im zweiten Abschnitt möchte ich die Bestimmungsfaktoren der verschiedenen Formen wirtschaftlicher Transaktionen erläutern und einige Ueberlegungen zur volkswirtschaftlichen Auswirkung der einzelnen Formen anstellen. Im dritten Abschnitt folgt dann eine kurze Charak- 
terisierung der internationalen Verflechtung der Schweiz. Dort wird insbesondere das Engagement der schweizerischen Chemischen Industrie in Entwicklungslăndern in Form von Joint Ventures genauer betrachtet; gleichzeitig erfolgt auch ein monetărer Vergleich des Umfanges der schweizerischen Entwicklungszusammenarbeit i.e.S. mit demjenigen der privatwirtschaftlichen Verflechtung der Schweiz in diesen Lăndern. Im vierten Abschnitt folgen einige Schlussfolgerungen. Dort wird erstens die Notwendigkeit von institutionellen Reformen in Entwicklungslăndern erläutert; erst dadurch ist mit einem vermehrten und adăquaten Know-how-Transfer in diese Lănder zu rechnen. Zweitens werden daraus auch einige Folgerungen für die Schweizer Entwicklungspolitik abgeleitet.

\section{Formen des Technologietransfers: die Transaktionsformen}

\subsection{Was Ist Technologietransfer?}

In der ökonomischen Theorie ist vereinfacht der gesamtwirtschaftliche Output (Y) eine Funktion des Einsatzes von Kapital (Geldeinheiten), Arbeit (Stunden) und Rohstoffen (Natur): $Y=f(A, K, N)$. Der funktionale Zusammenhang zwischen den Produktionsfaktoren und dem Output wird durch die vorhandene Produktionstechnologie und damit die Produktionsfunktion (f) ausgedrückt. Die gesamtwirtschaftliche Produktivität eines Landes misst, wieviel Output pro eingesetzte Arbeitsstunde ( $Y / A=$ Arbeitsproduktivität) oder eingesetzte Kapitaleinheit (Y/K = Kapitalproduktivität) erarbeitet wird. Ein technischer Fortschritt bedeutet nun, dass mit gleichviel Ressourcen (K,A,N) ein höherer gesamtwirtschaftlicher Output erarbeitet werden kann; der Output pro Kopf und damit das Pro-Kopf-Einkommen steigt. In diesen Modellen bestimmt das Technologieniveau eines Landes die gesamtwirtschaftliche Produktivität. Die sogenannte Technologielücke ăussert sich dadurch, dass die Produktivität eines Landes tiefer ist als diejenige eines anderen Landes.

Obwohl man oft nur dann von Technologietransfer spricht, wenn eine neue und bessere Maschine zur Herstellung von bestimmten Produkten von einem Land in das andere verschoben wird - also eine Mischung von Produktionsfaktor (Kapital) und Produktionstechnologie (technischer Fortschritt) -, so ist eine Einschränkung auf 'produktive' wirtschaftliche Aktivitäten (im Sinne der Herstellung von physischen Gütern) wenig sinnvoll und zu eng. Auch der Transfer von kaufmännischen, künstlerischen und anderen Fähigkeiten făllt darunter, insbesondere Produkte- und Marktknow-how oder auch internationale Dienstleistungen im Banken- oder Versicherungssektor. Es handelt sich also nicht nur um einen Technologietransfer, wenn eine hoch entwickelte Textilmaschine, verschiedene Bauteile eines schlüsselfertigen Elektrizitätswerkes oder eine einfache Handpumpe zur Wasserbeförderung die Schweizer Grenze mit Zielland Indien, Brasilien oder Madagaskar überqueren. Dazu gehört auch die von einem Schweizer Importeur erstellte Produktespezifikation für mögliche Importprodukte, ein 
Dienstleistungsexport in Form eines Beratungspaketes für die Etablierung eines Bankensystems, die Ausfuhr eines neuen Saatgutes oder eines speziellen chemischen Zwischenproduktes oder ein DEH-Mitarbeiter, ${ }^{1}$ der den Aufbau eines Bewăsserungssystems betreut.

\section{Figur 1: Verschiedene Beispiele von Technologietransfer}

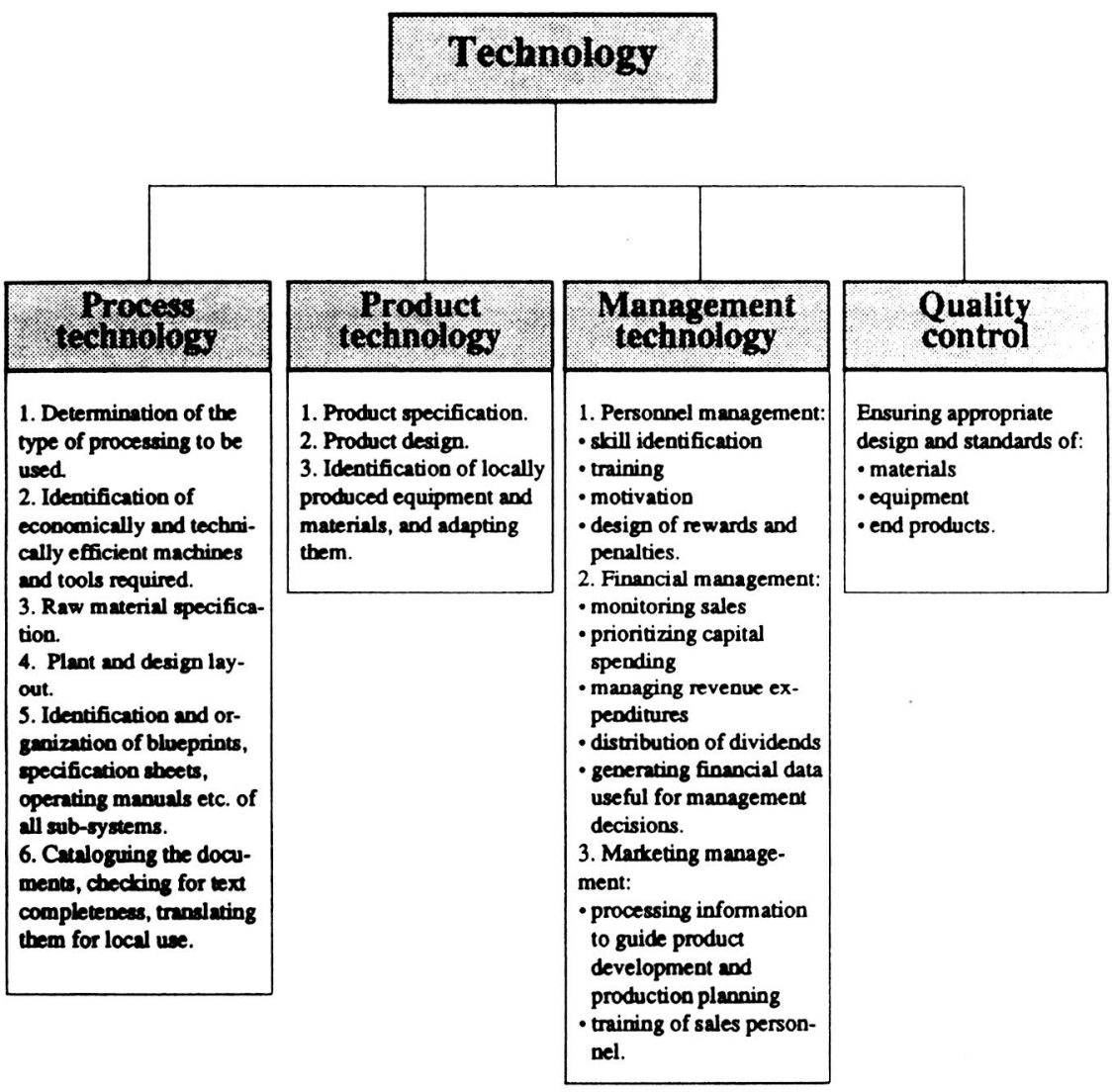

\section{Quelle}

United Nations (1988), Transnational Corporations in World Development, S. 178

1. $D E H=$ Direktion für Entwicklungszusammenarbeit und humanitäre Hilfe. 
In der Realität wird praktisch jede internationale Transaktion einen Technologie- bzw. Know-how-Transfer beinhalten. Erstens ist nămlich ein Transfer von Produktionsfaktoren oft mit einer bestimmten Technologie verbunden; dies ist z.B. typisch bei einer Direktinvestition. Hier wird neben dem Produktionsfaktor Kapital umfassendes Know-how (Maschinen, Produktionsprozesse, Managementtăhigkeiten etc.) transferiert. Kein Technologietransfer findet statt, wenn reines, ungebundenes Kapital z.B. in Form von Krediten transferiert wird. Zweitens verwischt sich der Unterschied zwischen Produktionsfaktoren und Gütern (Endprodukte, Enddienstleistungen). Viele exportierte Güter werden im Zielland wieder als Zwischenprodukte bzw. Produktionsfaktoren weitervenwendet. Auch ist mit einem Export von Konsumgütem ein Know-how-Transfer verbunden, da die Zusammensetzung des Gutes wiedenum als Produkt- und Produktionsknowhow für einen lokalen Produzenten dienen kann. Drittens zeigen die Ueberlegungen, dass wir besser den Begriff 'Know-how-Transfer' venwenden wollen, da er den Transfer sămtlichen Wissens beinhaltet und sich nicht auf die rein produktionsmässigen Fähigkeiten beschrănkt; denn gerade Wissen für die Beantwortung der Frage, welche Produkte wie und für wen produziert werden können und sollen, fehlt den potentiellen Unternehmern in Entwicklungsländern oft.

\subsection{Formen des Know-how-Transfers}

Ob Technologie oder Know-how innerhalb einer multinationalen Unternehmung transferiert wird, über einen Lizenzvertrag oder einen eingesetzten DEH-Mitarbeiter ins Zielland gelangt, ist ökonomisch eine Frage der Transaktionsform. Dabei gehe ich im folgenden nicht von der üblicherweise vorgenommenen Zweiteilung in 'Markt' und 'Hierarchie' aus, sondem ordne die in der Realität zahlreichen wirtschaftlichen Transaktionsformen nach den drei Typen 'Markt', 'Kooperation' und 'Hierarchie' (Integration).

Reine Markttransaktionen sind dadurch charakterisiert, dass unabhängige Wirtschaftssubjekte einen vollständig definierten Vertrag über den Inhalt des zu transferierenden Gutes abschliessen. Dabei besteht zwischen den Transaktionspartnern weder eine Bindung für künftige weitere Geschäfte, noch eine persönliche Beziehung; im Extremfall kann das Geschăft anonym abgewickelt werden. Typische Beispiele sind Börsengeschäfte (Wertpapiere, Rohstoffe), das klassische Tauschgeschäft auf dem Gemüsemarkt oder der Export von Agrarprodukten und Autos.

Hierarchisch koordinierte wirtschaftliche Aktivitäten werden innerhalb einer Unternehmung durchgeführt. Anstelle des Koordinationsinstrumentes 'Preis' wie bei der Markttransaktion - tritt hier das Autoritätsprinzip. Die Koordination wird durch eine übergeordnete, zentrale Leitung ausgeübt. Die Transaktionspartner sind nicht mehr unabhängig und stehen damit auch nicht mehr in einem antagonistischen Spannungsverhältnis wie bei der Markttransaktion; sie werden eingebunden in ein System mit klaren Spielregeln und Sanktionsmechanismen. Typische Beispiele hierzu sind sämtliche innerhalb einer Unternehmung abge- 
wickelten Transaktionen, im internationalen Rahmen die Direktinvestitionen durch multinationale Unternehmungen oder vom Ausland diktierte, zweckgebundene Entwicklungshilfe.

Zwischen diesen Extremvarianten wirtschaftlicher Transaktionen besteht ein dritter Typ, den wir Kooperation nennen wollen. Die wirtschaftliche Kooperationsform ist dadurch charakterisiert, dass die Transaktionspartner zwar unabhăngig bleiben, aber ein bestimmtes Mass an Bindung für künftiges Verhalten eingehen. Sie schliessen Vertrăge $a b$, die wegen der lăngerfristigen Perspektive unvollstăndig sind und dadurch auf beiden Seiten ein erhebliches Mass an Trittbrettfahrer-Verhalten zulassen. Als Beispiele dazu können Equity oder vertragliche Joint Ventures, aber auch Know-how- und Managementvertrăge aufgeführt werden.

In Figur 2 sind die erwăhnten Transaktionsformen dargestelt und den drei Transaktionstypen zugeordnet. Die unscharfen Grenzen zwischen den drei

\section{Figur 2: Das Transaktionsformen-Typenband}

\section{'Markt-Kooperation-Hierarchie'}

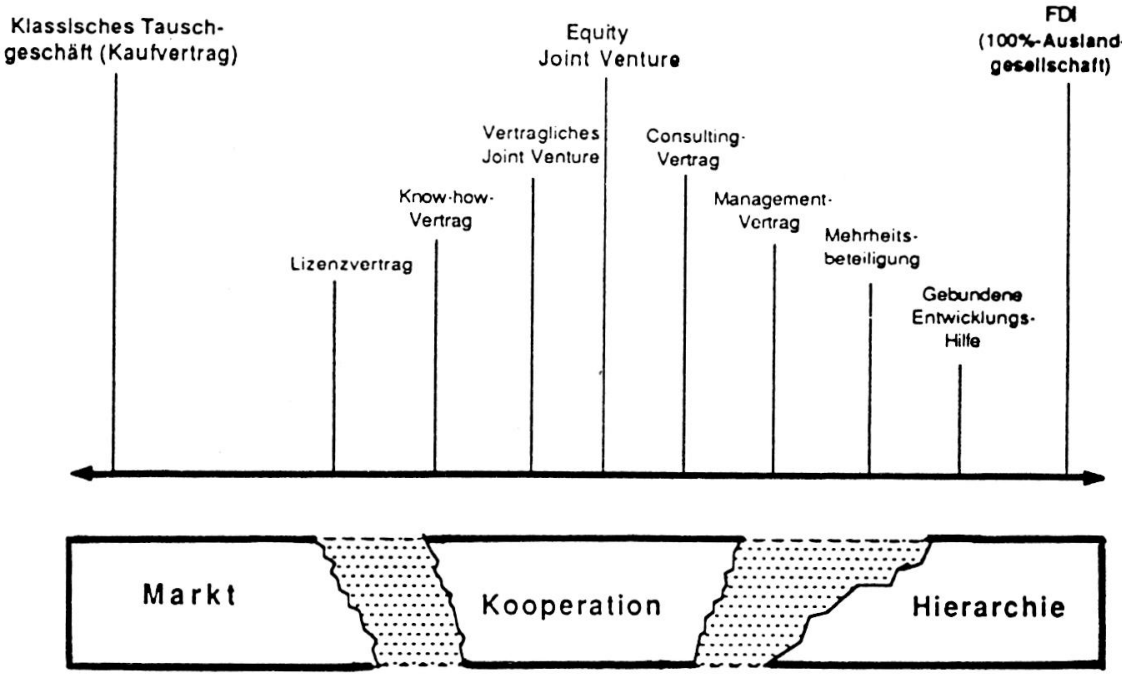


Typen sollen signalisieren, dass die Zuordnung einzelner Transaktionsformen manchmal arbitrăr bleibt. ${ }^{2}$

Die im obigen Typenband eingeordneten Transaktionsformen kőnnen wir folgendermassen charakterisieren:

(1) Internalisierung/Externalisierung: die Transaktionsformen zeichnen sich von links nach rechts durch eine zunehmende Internalisierung der Verwertung von unternehmungsspezifischen Ressourcen aus; d.h. wăhrend mittels eines Lizenzvertrages die produktionsmăssige Verwertung von technischem Wissen an den (externen) Lizenznehmer übertragen wird, findet die Nutzung des Wissens durch die Uebertragung an die Tochtergesellschaft firmenintern statt.

(2) Risiko der Verwertung: die Transaktionsformen zeichnen sich von links nach rechts durch eine zunehmende Verlagerung des Risikos vom Empfänger zum Absender des Gutes aus. Bei den marktmăssigen Transaktionsformen trăgt primăr der Empfänger das (Verwendungs)Risiko des transferierten Gutes (Einsatz des Zwischenproduktes, Verwendung des Knowhows). Bei den Kooperationsformen entsteht eine Risikoteilung, indem die Partner gemeinsam - eventuell in unterschiedlichem Ausmass - das Risiko der Wissensverwertung tragen (Produktion oder Vertrieb eines Produktes im Joint Venture). Bei den hierarchischen Transaktionsformen trăgt der Besitzer und Venwerter das Risiko alleine (Muttergesellschaft einer 100\%Tochtergesellschaft im Ausland, verantwortliche institution eines Entwicklungsprojektes).

(3) Kontrolle über Venwertung: die Transaktionsformen zeichnen sich von links nach rechts durch eine zunehmend verlagerte Verwertungskontrolle vom Empfänger zum Absender des Gutes aus: Verwendung des Zwischenproduktes durch den Kăufer (Markttransaktion), aufgeteilte Führungsverantwortung im Joint Venture (Kooperation), Lenkung der Investitionsmittel durch das Mutterhaus einer multinationalen Unternehmung oder durch eine Entwicklungsorganisation (Hierarchie).

(4) Kooperatives Verhalten: die Transaktionsformen zeichnen sich von links nach rechts dadurch aus, dass ab der Markttransaktion das für das Zustandekommen einer Transaktion notwendige kooperative Verhalten kontinuierlich ansteigt und beim Equity Joint Venture sehr ausgeprägt ist, um dann wieder zu sinken, je mehr man sich der hierarchischen Koordinationsstruktur nähert. Bei der Markttransaktion braucht es kein kooperatives Verhalten, weil der Vertrag vollständig ist und davon abweichendes Verhalten gerichtlich sanktioniert werden kann; bei der hierarchischen Koordinationsstruktur bestehen interne Sanktionsmechanismen; bei der Kooperation kommt we-

2. Natürlich wurden hier nicht alle möglichen Transaktionsformen eingeordnet, wie z.B. Turnkey, Barter, Junktim, Franchising etc.; die einzelnen Formen werden in der Realität zum Teil auch kombiniert (z.B. Joint Ventures und Lizenzverträge). 
gen der Unvollstăndigkeit der Vertrăge eine erfolgreiche Transaktion nur bei kooperativem Verhalten zustande.

\section{Bestimmungsfaktoren und Wirkungen der Transaktionsformen}

\subsection{Bestimmungsfaktoren der Wahl spezlfischer Transaktionsformen}

Warum wird Wissen, Know-how oder Information in der Realităt mit den genannten verschiedenen Transaktionsformen transferiert? Weshalb exportieren Unternehmungen Farbstoffe, Pharmazeutika oder Textilmaschinen als Fertigprodukte von einem Land in das andere, wăhrend sie in scheinbar ăhnlichen Făllen eigene Produktions- oder Vertriebsgesellschaften im Ausland erstellen? Weshalb wăhlen wiederum andere Firmen den Weg der Internationalisierung durch Kooperationsformen wie Lizenz-, Know-how-Vertrăge oder Joint Ventures? Oder weshalb verkauft die DEH ihr Wissen nicht an Personen in Entwicklungslăndern, sondern sendet eigene Mitarbeiter dorthin, die das Wissen selber umsetzen?

Waren früher vor allem imperialistische oder zumindest marktmacht-orientierte Erklărungsansătze verbreitet, so dominieren heute in der ökonomischen Theorie - meines Erachtens zurecht - die Transaktionskosten-Ansătze. Diese auf Coase (1937) zurückgeführten Ueberlegungen erklăren die simultane Existenz von Firmen und Märkten aufgrund von Transaktionskosten. ${ }^{3}$ Die Frage der Verwertung von Wissen wird deshalb bedeutsam, weil der Ertrag von Informationen nur beschränkt direkt über den Markt angeeignet werden kann; so schrieb Richardson : "(...) technology is a very special commodity and the market for it a very special market. ${ }^{\text {". }}$ Nicht patentierbares Know-how zum Beispiel ist in der Realität sehr schwierig marktmässig handelbar (z.B. Organisationsgeschick, Marketingfăhigkeiten, Software); dies führt dazu, dass Unternehmungen die Verwertung internalisieren und so ein entgeltloses Abfliessen des Knowhows verhindern. Die relativen Kosten der Transaktion von Wissen entscheiden also darüber, welche Transaktionsform im konkreten Fall gewăhlt wird. Dazu gehören insbesondere die Vertragsabschluss- und Vertragskontrollkosten bei der Markttransaktion oder die Organisations- und Umsetzungskosten bei der firmeninternen Know-how-Verwertung.

Aufgrund dieser hier skizzierten Theorie lässt sich folgendes aussagen. ${ }^{5}$ Die verschiedenen Transaktionsformen sind das Resultat von natürlichen Unvoll-

3. R.H. Coase (1937), The Nature of the Firm, in: Economica, Vol. 4, No. 4, S. 386 405.

4. G.B. Richardson (1972), The Organisation of Industry, in: the Economic Journal, September, S. 893.

5. Für eine eingehende Darstellung vergleiche man R. Weder (1989), Joint Venture, Grüsch: Rüøgger. 
kommenheiten der Know-how-Märkte. In der Realităt bestehen allerdings zahlreiche künstliche Marktunvollkommenheiten bzw. Markteingriffe, die bestimmte internationale Transaktionsformen behindern und andere begünstigen. Dadurch wird auch die länderspezifische Anordnung von wirtschaftlichen Aktivităten beeinflusst. Dazu nur einige Beispiele:

(1) Zölle: Importzölle auf Fertigproduktexporte begünstigen die Produktionsverlagerung, wobei die Zollstruktur bestimmt, welche Schritte innerhalb der Produkteherstellkette verlagert werden; d.h. hohe Importzölle auf Zwischenprodukten begünstigen die lokale Herstellung von Zwischenprodukten, behindern aber gleichzeitig diejenige der Fertigprodukte.

(2) Keine Garantie von Patenten: Können keine Eigentumsrechte an patentiertem Know-how garantiert werden, so werden sowohl inländische Erfindungen und Innovationen als auch Verlagerungen von Forschungs- und Entwicklungstätigkeiten durch ausländische Unternehmungen behindert. Des weiteren erhalten Markt- und Kooperationsformen (z.B. Lizenzverträge) einen relativen Nachteil.

(3) Unsicheres Vertragsrecht. Werden Vertragsverletzungen nicht oder ungenügend geahndet und herrscht also generell eine hohe institutionelle Unsicherheit, so haben sämtliche marktmässigen oder marktähnlichen Transaktionsformen einen Nachteil (z.B. Kaufvertrag, Lizenzvertrag, Know-howVertrag); opportunistische Transaktionspartner werden also Vertrăge kaum einhalten und das erhaltene Know-how unbeschrănkt und unkontrolliert weiterverwenden können. Die Firmen dürtten den Know-how-Transfer deshalb in firmeninterne Transaktionen (Venwertung in einer eigenen Tochtergesellschaft) oder dann in langfristige Kooperationsformen aufgrund einer Vertrauensbasis zum ausländischen Partner (z.B. Joint Venture) einbinden.

(4) Steuern auf Royalties: Steuern auf (innerbetrieblichen) Verrechnungen von Know-how-Transfers (Forschung \& Entwicklung, Managementunterstützung etc.) behindern eine Verlagerung von Produktionsschritten gemäss den komparativen Vorteilen. Multinationale Unternehmungen werden einen Grossteil der Produktion im Mutterland bewahren, um via überhöhte Zwischenproduktpreise den Know-how-Transfer zu finanzieren.

\subsection{Auswirkungen von einzelnen Transaktionsformen}

Bezüglich der Wirkungsanalyse verschiedener internationaler Transaktionsformen bewegen wir uns auf relativ schwachem Grund. Die normative Aussenwirtschaftstheorie behauptet zwar, dass sowohl der freie Handel von Gütern als auch die international freie Mobilität von Produktionsfaktoren die Gesamtallokation von Ressourcen verbessert und somit die Gesamtwohlfahrt aller Länder erhöht. Wie wir gesehen haben, können Direktinvestitionen, Lizenzverträge oder Joint Ventures als in bestimmten Situationen effizientere Formen des internationalen Transfers von Know-how, Produktionsfaktoren oder Gütern betrachtet 
werden. Deshalb gilt die Aussage der Theorie grundsătzlich für alle Formen internationaler Transaktionen.

Diese Folgenung in die Realităt zu übertragen, ist allerdings nur beschrănkt möglich. Erstens macht die Theorie keine verbindliche Aussage darüber, wie die aus den internationalen Transaktionen entstehenden Wohlfahrtsgewinne auf die Lănder und auf einzelne Sektoren innerhalb eines Landes verteilt sind. ${ }^{6}$ Zweitens werden in der Theorie Annahmen über Mărkte und Marktverhalten gemacht, die in der Wirklichkeit oft nicht oder nur teilweise gelten (zum Beispiel bestehen oft grosse Strukturanpassungskosten, Preisrigidităten, oligopolistische Marktstrukturen oder Bargainingsituationen). ${ }^{7}$ Somit begnügen wir uns also mit ein paar grundsătzlichen Ueberlegungen über die Auswirkungen der verschiedenen Transaktionsformen auf das Zielland.

Marktmässige oder marktăhnliche Formen des internationalen Know-howTransfers haben den Nachteil, dass ein breites Spektrum von Know-how in dieser Form kaum oder nicht übertragbar ist (z.B. Produkt- und Markt-Know-how, Marketingfähigkeiten, Organisationsfähigkeiten). Des weiteren verlangt diese Art von Transaktion (z.B. ein Lizenzvertrag) eine relativ grosse Vertragssicherheit und klare Eigentumsrechte in Form von Patentschutz, die in vielen Entwicklungsländern nicht vorhanden sind. Schliesslich liegt das Verwertungsrisiko und die -kontrolle vollstăndig beim Know-how-Empfănger, was bei vorhandener hoher Wissensasymmetrie der Transaktionspartner ein Nachteil ist. Auch ist zu enwarten, dass die Marktransparenz der Kăufer von (differenzierten) Importprodukten in den Entwicklungsländern relativ gering ist, was zu monopolistischem Spielraum der Exporteure führt. ${ }^{8}$

Hierarchische Transaktionsformen haben wiederum folgende nachteilige Wirkungen: ${ }^{9}$ Direktinvestitionen sind relativ kapitalintensiv und haben deshalb keine grossen Beschäftigungseffekte in den Entwicklungslăndern. ${ }^{10}$ Dies liegt

6. Man könnte auch sagen, dass die möglichen Wirkungen auf die Verteilung des Gewinnes durch die Annahme bzw. Forderung von Kompensationszahlungen geschickt eliminiert wurden, was - wie die politische Oekonomie zeigt - verfehlt ist.

7. Diese Abweichungen bedeuten, dass für eine praktische Wirkungsanalyse verschiedener Formen des internationalen Know-how-Transfers zuerst ein auf alle Länder anwendbares Analysekonzept zu erarbeiten wäre; dieses müsste dann mit empirischen Daten gefüllt werden, was hoffentlich pro Transaktionsform zu Gemeinsamkeiten in der Wirkung führen wird. Von einem solchen Konzept und dieser Analyse sind wir allerdings noch weit entfernt.

8. Vgl. auch die interessanten Analysen der Weltbank, die im Fall von Afrika zu einem ähnlichen Schluss kommt: A.J. Yeats (1990), Zahlen afrikanische Länder mehr für Importe?, in: Finanzierung \& Entwicklung, Juni, S. 38-40.

9. Vgl. dazu auch die Aussagen und Quellenhinweise in United Nations, a.a.O., S. $180 \mathrm{ft}$.

10. Ich spreche hier vor allem von schweizerischen multinationalen Unternehmungen und nicht von den sogenannten resource-based Multis (Plantagen, Eisenerzextraktion, Förderung von Kohle, Gold oder Erdöl etc.). 
daran, dass auslăndische Firmen einen leichteren Zugang zu Kapital haben und Entwicklungslănder über wenige ausgebildete Arbeitskrăfte verfügen. Direktinvestitionen beinhalten meist hoch entwickelte Technologien und haben - auch wegen der Notwendigkeit qualitativ hochstehender Vorprodukte - wenige Folgeeffekte auf andere Firmen und Industrien im Zielland (Fördenung eines Dualismus). Dass Firmen Direktinvestitionen in Form von 100\%-Tochtergesellschaften wăhlen, liegt insbesondere an den durch die Verlagerung der gleichen Technologie entstehenden economies of scale sowie an den beim alfălligen Reexport zu erfüllenden weltweiten Standards multinationaler Unternehmungen. Da das Risiko und die Kontrolle über die Verwertung des transferierten Know-hows vollstăndig bei der auslăndischen Muttergesellschaft liegt, wird die lokale Aktivităt in die weltweite Unternehmungsstrategie eingebettet, was nicht immer von Vorteil für die wirtschaftliche Entwicklung des Gastlandes sein muss. In ähnlicher Weise stehen auch Entwicklungsprojekte unter der Kontrolle des Auslandes. Dabei ist hier das Risiko in Anbetracht der nicht geforderten Wirtschaftlichkeit unbedeutend. Art, Umfang und Einsatzort des Technologietransfers hängen aber wesentlich von den Zielsetzungen bzw. Ideologien der Akteure im Ursprungsland ab. ${ }^{11}$

Internationale Kooperationsformen haben gegenüber den oben erwähnten Transaktionstypen gewisse Vorteile. Es ist zu erwarten, dass Joint Ventures mit einem arbeitsintensiveren und weniger hochentwickelten Technologietransfer verbunden sind. Dies liegt an der für diese Formen typischen Gefahr, dass Know-how unentgeltlich abfliesst; deshalb werden keine neuesten Technologien in diese Formen hineingebracht. Auch besteht die Notwendigkeit, dass der Partner im Entwicklungsland komplementäre Făhigkeiten einbringt; dies kann er oft nur, wenn er die Technologie versteht und so selber weiterentwickeln kann. Die Spillover-Effekte auf andere Industrien des Ziellandes dürtten deshalb grösser sein. Des weiteren trăgt der lokale Partner sowohl das Venwertungsrisiko und die Verwertungskontrolle mit, was zu einer motivierenden Verhaltensstruktur führt. Schliesslich können internationale Kooperationsformen deshalb auch weniger in das globale Netz des auslăndischen Partners eingefügt werden, was die Exportchancen aus dem Kooperationsabkommen erhöht. Vielversprechend erscheinen auch Vertriebs-Joint Venture mit Sitz in industrialisierten Lăndern, da diese Kooperationsform den Partnern in Entwicklungslăndern zu besseren Marktinformationen und damit Absatzmöglichkeiten verhelfen könnten.

11. In einer Analyse der Wirkungen von Entwicklungshilfe und Direktinvestitionen auf volkswirtschaftliche Aggregate zahlreicher Entwicklungsländer kommt Mahdavi zum Schluss, dass Entwicklungshilfe vor allem zu einer Erhöhung der Konsum- und Staatsausgabenquote führt, während Direktinvestitionen eine Erhöhung der Investitions- und Exportquote mit sich bringen; vgl. S. Mahdavi (1990), The Effects of Foreign Resouce Inflows on Composition of Aggregate Expenditure in Developing Countries, in: Kyklos, Vol. 43, No. 1, S. 111-134. 


\section{Die Internationale Verflechtung der Schweiz}

Nach diesen allgemeinen Ausführungen wollen wir einen Blick auf die Internationalisierung der Schweiz werfen und aufzeigen, wie verbreitet die verschiedenen Transaktionsformen in der Schweizer Wirtschaft sind. Vor diesem Hintergrund wird es nötig sein, die Entwicklungszusammenarbeit i.e.S. (Tătigkeit der DEH und privater Hilfswerke) mit den Aktivităten der Schweizer Wirtschaft in Entwicklungslăndern zu vergleichen. Dieser Vergleich wird zeigen, dass die monetăren Grőssenordnungen der privatwirtschaftlichen Verflechtung zu einer Relativierung der Bedeutung der Entwicklungszusammenarbeit i.e.S. führen. Mit anderen Worten, die Auswirkungen der Entwicklungszusammenarbeit i.e.S. dürtten - aggregiert betrachtet - in vielen Ziellăndern kleiner sein als diejenigen der Schweizer Wirtschaft.

Die Schweizer Wirtschaft weist im intemationalen Vergleich eine bezüglich Intensităt und Vielfalt ausgeprägte weltwirtschaftliche Verflechtung auf. Die Schweiz bezieht Platz 14 auf der Rangliste der grössten Weltexporteure von Waren (1988) und Platz 11 der führenden Exportlănder von Dienstleistungen (1987). ${ }^{12}$ Die Exportquoten - bei einer insgesamt negativen Handelsbilanz liegen auf vergleichbarem Niveau anderer industrialisierter Lănder. Eine einmalige Spitzenposition belegt die Schweiz bezüglich der Faktoreinkommen (Kapitaleinkommen in Form von Zinsen auf Dividenden aus Portfolio- und Direktinvestitionen, Lizenzeinnahmen, Zinsdifferenzgeschăft der Banken sowie Arbeitseinkommen); die Nettofaktoreinkommen (Saldo der Faktoreinkommen) machten 1988 knapp 5\% des Bruttosozialproduktes aus, 1985 waren es 5,5\%. Dieser Prozentsatz liegt weit über demjenigen von anderen industrialisierten Lăndern. ${ }^{13}$

Die führende Position im Nettofaktor-Einkommensbereich beruht unter anderem auch darauf, dass die Schweiz über einen hohen Bestand an Direktinvestitionen im Ausland verfügt; 1986 lag sie mit einem Bestand von SFr. 55,5 Mrd. an achter Stelle in der Rangliste der weltweit grössten Direktinvestoren, 1978 war es sogar Rang fünf. ${ }^{14}$ Die Bedeutung der Direktinvestitionen für die schweizerische Volkswirtschaft wird auch daran deutlich, dass die Schweizer Direktinvestoren 1988 891'400 Personen in auslăndischen Betriebsstătten beschăftigten, was etwa einen Viertel der gesamten Inlandbeschăftigung ausmacht. ${ }^{15}$

Schliesslich zeigen diese Zahlen über die aussenwirtschaftliche Verflechtung der Schweiz implizit, dass die schweizerische Wirtschaft von all den oben

12. Vgl. Gatt (1989), International Trade 1988-89, Genf.

13. Vgl. dazu C. Knöpfel (1988), Der Einfluss der politischen Stabilität auf die internationale Wettbewerbsfähigkeit der Schweiz, Grüsch, S. 19; die BRD wies 1985 einen Prozentsatz von 0.4 auf, USA von 1.0, England von 0.7 oder Schweden von -2.6.

14. Vgl. S. Borner/F. Wehrle (1984), Die Sechste Schweiz, Zürich, S. 79 sowie H. Krägenau (1987), Internationale Direktinvestitionen, Hamburg, S. 361.

15. Vgl. SNB (1989), Geld, Währung und Konjunktur, Quartalsheft No. 43, S. 370. 
enwăhnten Transaktionsformen regen Gebrauch macht. Zwar fehlen offizielle Zahlen über das Ausmass von zum Beispiel den Ertrăgen aus Lizenzvertrăgen, doch dürtten sie gemäss inoffiziellen Schătzungen mehrere hundert Millionen SFr. ausmachen. Auch was den Abschluss von Joint Ventures im Ausland anbelangt, gibt es keine konkreten Informationen. Einzig für die schweizerische Chemische Industrie wurde 1988 eine empirische Untersuchung durchgeführt, die hervorbrachte, dass 1986 etwa 80 Joint Ventures im Ausland bestanden. ${ }^{16}$

Ohne hier auf die geographische Verflechtung im Besonderen einzugehen, kann gesagt werden, dass die meisten wirtschaftlichen Transaktionen der Schweizer Wirtschaft mit den anderen industrialisierten Lăndern bestehen. Trotzdem gingen 1988 nicht weniger als 17\% der Schweizer Warenexporte in die Entwicklungsländer (inkl. OPEC-Staaten und Schwellenländer), 8\% der Importe kamen aus diesen Ländern. Die Schweiz erzielte damit mit den Entwicklungslăndern einen Handelsbilanzüberschuss von knapp 6 Mrd. SFr., wăhrend die gesamte Handelsbilanz bekanntlich ja einen negativen Saldo ausweist. ${ }^{17} \mathrm{Be}-$ züglich der Direktinvestitionen weist die Bilanz der Schweiz in diesen Lăndern einen Auslandbestand von 11,2 Mrd. SFr. aus (1988), was $16 \%$ der gesamten Direktinvestitionen ausmacht. ${ }^{18}$ Wiederum fehlen Daten über die Verbreitung anderer Transaktionsformen; immerhin hat die oben erwähnte Studie gezeigt, dass sich 1986 von allen Ausland-Joint Ventures der schweizerischen Chemischen Industrie etwa $40 \%$ in den Entwicklungsländern (inkl. Ostblock) befanden.

In Figur 3 ist die geographische Verteilung aller Joint Ventures der schweizerischen Chemischen Industrie (1986) dargestellt. Der grösste Teil der Joint Ventures in Entwicklungsländern entfällt dabei auf die lateinamerikanischen und die ostasiatischen Schwellenländer (10 bzw. 8 Joint Ventures). Interessanterweise folgen aber sehr schnell Indien und Pakistan (7) sowie Afrika (7), wobei sich die "Afrikanischen Joint Ventures" auf die Länder Ägypten, Kenia, Nigeria und Marokko beschränken. Schliesslich folgen Joint Ventures in weiteren Lăndern Ostasiens (3), in den arabischen Ölexport- (3) sowie in den Ostblockländern. Vor allem in den letzteren dürten die kürzlichen politischen und ökonomischen Veränderungen den Bestand der (geplanten) Joint Ventures stark erhöht haben.

Dabei umfassen die Joint Ventures in Entwicklungsländern ein breites Spektrum von Aktivitäten und Strukturen. Dazu gehören beispielsweise Joint Ventures zur Produktion von Bauchemikalien (Afrika, Lateinamerika), zur Herstellung und zum Vertrieb von Pharmazeutika (Lateinamerika, Südostasien, Indien) wie auch zur Produktion und Formulierung von Textilfarbstoffen (Indien, Lateinamerika). Bei Vertriebs-Joint Ventures bringen lokale Partner vor allem das Markt-Know-how und das Vertriebsnetz in das Kooperationsabkommen ein. Es sind aber auch Produktions-Joint Ventures in Entwicklungsländern verbreitet,

16. Vgl. R. Weder (1989), a.a.O., S. 209.

17. Vgl. Eidgenössische Oberzolldirektion (1988), Schweizerische Aussenhandelsstatistik, Jahresbericht 1988, Bern, S. 92.

18. SNB (1989), a.a.O., S. 371. 
wobei hier nicht selten ein Joint Venture mit einem Partner bzw. Konkurrenten aus einem Industrieland abgeschlossen wurde (z.B. Ciba-Geigy und Sandoz auf dem Farbstoffgebiet in Brasilien). Es ist zu erwarten, dass letztere Joint Ventures einen Verdrăngungs-Effekt auf lokale Investitionen sowie auf Importe haben - sie waren aber selber eine Folge der (gescheiterten) Importsubstitutionspolitik lateinamerikanischer Lănder. Kaum vorzufinden sind Joint Ventures im F\&E-Bereich, weil für solche Aktivităten sowohl der Ausbildungsstand als auch die Garantien von Eigentumsrechten in Entwicklungslăndern oft ungenügend sind. Erst langsam im Ansteigen begriffen sind exportorientierte Joint Ventures, vor allem in den ostasiatischen und lateinamerikanischen Schwellenlăndern und beschrănkt auf relativ standardisierte, einfache Produkte.

Figur 3: Geographische Verteilung der Joint Ventures der schweizerischen Chemischen Industrie (1986)

\begin{tabular}{|c|c|c|c|c|}
\hline \multirow[t]{2}{*}{$\begin{array}{l}\text { Standort der } \\
\text { Joint Ventures }\end{array}$} & \multicolumn{2}{|c|}{$\begin{array}{l}\text { Anzahl Joint } \\
\text { Ventures } \\
\text { pro Standort }\end{array}$} & \multicolumn{2}{|c|}{$\begin{array}{l}\text { Anzahl Firmen mit } \\
\text { Joint Ventures } \\
\text { pro Standort }\end{array}$} \\
\hline & abs. & $\%$ & abs. & $\%$ \\
\hline Schweiz & 21 & 20 & 9 & 45 \\
\hline EG & 18 & 17 & 7 & 35 \\
\hline Japan & 12 & 11 & 3 & 15 \\
\hline Lateinam. NICs* & 10 & 10 & 3 & 15 \\
\hline Ostasiat. NICs** & 8 & 8 & 4 & 20 \\
\hline USA/Kanada & 7 & 7 & 5 & 25 \\
\hline Indien/Pakistan & 7 & 7 & 3 & 15 \\
\hline Afrika*** & 7 & 7 & 3 & 15 \\
\hline Übriges Westeuropa & 3 & 3 & 3 & 15 \\
\hline Übriges Ostasien & 3 & 3 & 2 & 10 \\
\hline Arab. Ölexportlănder & 3 & 3 & 3 & 15 \\
\hline Osteuropa/UdSSR & 2 & 2 & 2 & 10 \\
\hline Australien/Neuseeland & 1 & 1 & 1 & 5 \\
\hline Übriges Lateinamerika & 1 & 1 & 1 & 5 \\
\hline China & 0 & 0 & 0 & 0 \\
\hline TOTAL & 103 & 100 & 20 & 100 \\
\hline \multicolumn{5}{|c|}{$\begin{array}{l}\text { * Argentinien, Brasilien, Mexiko, Venezuela } \\
* * \text { Hongkong, Singapur, Taiwan, Südkorea } \\
\text { *** Ägypten, Kenia, Nigeria, Marokko }\end{array}$} \\
\hline \multicolumn{5}{|c|}{ Quelle: R. Weder (1989), S. 209} \\
\hline
\end{tabular}


Dieser kurze Ueberblick gibt einen Hinweis auf die hohe und vielfăltige internationale Verflechtung der Schweizer Wirtschaft und zeigt auf, dass die wirtschaftlichen Beziehungen der Schweiz mit der Dritten Welt - inklusive OPECund Schwellenlănder - nicht etwa nur eine Randerscheinung sind. Steltt man neben den Handelsbilanzüberschuss von 6 Mrd. SFr. (1988) oder neben die Zunahme der Schweizer Direktinvestitionen von etwa 3 Mrd. SFr. im Jahre 1988 die jăhrlichen Ausgaben der offentlichen und privaten Schweizer Entwicklungszusammenarbeit von knapp 1 Mrd. SFr. (1988: 987 Mio), so wird folgendes klar: bei einer Beurteilung der Wirkungen der wirtschaftlichen Transaktionen der Schweiz auf die Entwicklungslănder können nicht nur die entwicklungspolitischen Massnahmen i.e.S. als Grundlage genommen werden. Die Grössenordnung der frei ablaufenden, privatwirtschaftlichen Transaktionen mit diesen Lăndern verlangt nach einer übergeordneten, integrierten Betrachtung. Insbesondere wird in diesem Zusammenhang aber auch die Trennung von Aussenwirtschafts- und Entwicklungspolitik höchst problematisch und fragwürdig. Es stellen sich relativ klar zu beantwortende Fragen, wie zum Beispiel die folgende: "würde ein Land wie die Schweiz nicht weit mehr zur Entwicklung im Ausland beitragen, wenn es den Entwicklungsländern vermehrt Exportchancen in $\mathrm{Be}$ reichen wie der Landwirtschaft einrăumen würde, damit auch diese Lănder wie die Schweiz die Möglichkeit haben, diejenigen Güter zu exportieren, in denen sie komparative Vorteile besitzen?"

\section{Schlussfolgerungen}

Ziehen wir nun einige Folgenungen für die wirtschaftlichen Reformen in den Entwicklungsländern sowie die Entwicklungspolitik in der Schweiz:

\subsection{Institutionelle Reformen In Entwicklungslăndern als Notwendigkeit}

Eine unter Oekonomen lange Zeit verbreitete Antwort auf die Frage nach den Ursachen der Unterentwicklung weist auf die unterschiedliche Ressourcenausstattung der einzelnen Länder hin: den unterentwickelten Lăndern fehle es vor allem an Investitionskapital, an ausgebildeten Arbeitskrăften (Humankapital) und - natürlich - an Technologie. Würde nămlich mehr investiert und zusătzlich noch die Kapitalproduktivität erhöht, so müsse in einer Volkswirtschaft auch die Wachstumsrate zunehmen. Die daraus abgeleitete Therapie lautete also: Erhöhung des Kapitalstockes durch Kapitaltransfer (Kredite, Schenkungen), Ausbildung der Arbeitskräfte durch den Aufbau von Schulen (beschleunigt durch Entwicklungsorganisationen) und Förderung des Technologietransfers (oft durch den Aufbau ausländisch finanzierter High-Tech-Projekte). 
Heute erscheint diese traditionelle Antwort weniger plausibel. ${ }^{19}$ Erstens müsste ja bei einer mangelnden Ressourcenausstattung eines Landes der private Kapital- und Technologietransfer vom Ausland überdurchschnittliche Renditen versprechen; ăhnlich müsste der Mangel an gut ausgebildeten Arbeitskrăften sowohl Inlăndern als auch Auslăndern hohe Lơhne versprechen. Aufgrund dieser Marktsignale würde deshalb ein Anreiz für den internationalen Transfer solcher Ressourcen entstehen. Eine internationale Angleichung der lănderweisen Ressourcenausstattungen und gesamtwirtschaftlichen Produktivităten wăre die natürliche Folge. Eine öffentliche Förderung des Transfers von Kapital und Know-how würde sich enübrigen, da der Markt dies selber besser und schneller tut. Nun ist es aber so, dass viele gut ausgebildete Arbeitskrăfte aus Entwicklungslăndern emigrieren und eine Beschăftigung in den industrialisierten Låndern suchen. Besteht also gar kein Mangel an Humankapital?

Zweitens zeigen aber auch die Erfahrungen der letzten Jahrzehnte, dass die von den industrialisierten Lăndern finanzierten oder zumindest grosszügig kreditierten internationalen Transfers von Kapital und Technologie in vielen Ländern zu keiner Erhöhung der Produktivităt und damit des Wohlstandes geführt haben - im Gegenteil. Ein Zeichen der mangelnden Zielkonformităt (staatlich mitfinanzierter) Ressourcentransfers sind die immer wieder bekannt gewordenen 'weissen Elefanten' - defizităre Grossprojekte - und ist auch die in den letzten Jahrzehnten entbrannte Debatte über die 'angepassten Technologien'.

Diese Ueberlegungen legen den Schluss nahe, dass nicht die mangelnde Ausstattung mit Ressourcen per se die primäre Ursache der Unterentwicklung der Zweiten, Dritten oder Vierten Welt ist. Vielmehr sind dafür noch andere, wichtigere volkswirtschaftliche Unterschiede verantwortlich. Neuere empirische Analysen über das Verhalten wirtschaftlicher und politischer Akteure in Entwicklungslăndern zeigen immer mehr, dass die wirtschaftlichen und politischen Systeme dieser Lánder bzw. deren Konstitutionen oder Institutionen erheblich von denjenigen der westlichen Industrielănder abweichen. Dabei ist die Aufteilung der Systeme in Plan- und Marktwirtschaften heute sicher zu einfach. Die Entwicklungslănder weisen aber eine signifikante Gemeinsamkeit auf bezüglich der Setzung oder eben 'Nicht-Setzung' von entwicklungsfördemden Verhaltensanreizen. So ist z.B. das peruanische Wirtschaftssystem durch eine überbordende und inkonsistente Regelungsdichte gekennzeichnet, die jede private Initiative unterbindet. De Soto kommt aufgrund einer neuen empirischen Studie zum Schluss, dass es den Peruanern nicht an unternehmerischen Făhigkeiten fehlt,

19. Vgl. J. Stiglitz (1989), Perspectives on Economic Development, AEA, P\&P, May, S. 197-203. 
sondern an einem System, das diese mobilisiert. ${ }^{20}$

Aufgrund der obigen Ausführungen lăsst sich zu dem hier gestellten Thema für die Entwicklungslănder und damit die Ziellănder des Know-how-Transfers folgendes aussagen:

(1) Eine notwendige Bedingung für die wirtschaftliche Entwicklung eines Landes ist also nicht seine Grundausstattung mit Ressourcen (Kapital, Arbeit, Technologie oder Rohstoffen). Dies kann zwar ein vorübergehender Vorteil sein, dürfte aber nicht von dauerhafter Wirkung auf die Entwicklung sein. Ein Paradebeispiel dazu ist die Schweiz.

(2) Eine wesentliche Voraussetzung für die wirtschaftliche Entwicklung ist vielmehr die Existenz eines politisch-ökonomischen Systems, das die Mobilität der Produktionsfaktoren innerhalb des Landes erhöht und so den effizienten Einsatz der Ressourcen maximiert, indem diese in die Sektoren mit der grössten relativen Produktivität geführt werden. Es geht - anders ausgedrückt - nicht primär um die Produktionsfaktor-Ausstattung, sondern um das Zusammenspiel und den Prozess des 'upgrading' der Faktoren. ${ }^{21}$ Oder in leichter Veränderung des berühmten Listschen Zitates: "wichtiger als der Reichtum an Produktionsfaktoren selber ist die systembedingte Möglichkeit, solche zu schaffen, zu verbessern und in die relativ ertragbringenderen Wirtschaftsbereiche zu investieren". 22

(3) Ob ein Know-how-Transfer auch die gesamtwirtschaftliche Produktivität erhöht, sich also im technischen Fortschritt niederschlägt und damit zu einem

20. De Soto zeigt überzeugend, dass sich in Peru infolge eines überregulierten und willkürlichen legalen bzw. formellen Wirtschaftssystems ein informeller Sektor gebildet hat, der nach marktwirtschaftlichen Prinzipien den unternehmerischen Initiativen freien Lauf lässt: Händler auf den Strassen, mehr als $90 \%$ des Transportgewerbes etc. Der legale Sektor hat das Entstehen des informellen zum Beispiel dadurch provoziert, dass das Erteilen einer Bewilligung für die Eröffnung eines Geschäftes 289 Tage davert sowie Gebühren in der Höhe des 32-Fachen des Minimallohnes beim Antragsteller verursacht. Allerdings muss dieser illegale Sektor, der nach durchaus legalen Zielen strebt, mit dem Nachteil fertig werden, dass keine Eigentumsrechte, keine Vertragsrechte, keine Gerichtsbarkeit und auch keine Sozialrechte garantiert sind. Starke Impulse auf die Entwicklung können deshalb vom informellen Sektor in dieser Form nicht erwartet werden; vgl. H. De Soto (1989), The Other Path. The Invisible Revolution in the World, N.Y.: Harper \& Row.

21. Mit 'upgrading' ist der Prozess der Verbesserung von vorhandenen Produktionstechniken, der laufenden Weiterbildung von technischen und kaufmännischen Mitarbeitern sowie die Realisierung von Erfindungen und Innovationen gemeint; vgl. dazu auch M. Porter (1990), Competitive Advantage of Nations, New York: McMillan.

22. List schrieb: "Die Kraft Reichtümer zu schaffen ist demnach unendlich wichtiger als der Reichtum selbst; sie verbürgt nicht nur den Besitz und die Vermehrung des Erworbenen, sondern auch den Ersatz des Verlorenen"; vgl. F. List (1844), Das nationale System der politischen Oekonomie, Stuttgart \& Tübingen. 
höheren Pro-Kopf-Einkommen führt, hăngt also von zahlreichen Faktoren ab. Sobald Marktunvollkommenheiten und Preisverzerrungen bestehen, ist dies sehr unwahrscheinlich. Der technische Fortschritt ist nicht automatisch gegeben, sondern hăngt wesentlich von einem funktionierenden Markt ab. Der hinter dem Begriff 'Technologietransfer' stehende Mythos ist somit beseitigt, der suggeriert, dass damit im Zielland automatisch eine Produktivitătserhöhung in bestimmten Sektoren verbunden sei.

(4) Die oben enwăhnten "hierarchischen" Transaktionsformen sind oft eine Folge von institutionellen Unsicherheiten in den Entwicklungsländern. In bestimmten Situationen an sich geeignete Transaktionsformen kommen so nicht zustande, weil eben Eigentumsrechte und Vertragssicherheiten nicht garantiert werden. Es werden deshalb entweder bestimmte Transaktionsformen gewăhtt (z.B. Direktinvestitionen), die geringere positive Wirkungen auf das Zielland haben, oder sie unterbleiben gänzlich.

Eine 'conditio sine qua non' für wirtschaftliche Entwicklung ist also ein politisch-wirtschaftliches System, das sowohl die eigenen Produktionsfaktoren in die Branchen mit komparativen Vorteilen leitet als auch attraktiv ist für international mobile Produktionsfaktoren. Insbesondere für die letzte Funktion sind Determinanten wie Rechtssicherheit, Eigentumsrechte oder politische Stabilität bedeutend.

\subsection{Neue Schwerpunkte der Schweizer Entwicklungs- und Aussenwirtschaftspolitik als Chance}

Aufgrund der gemachten Ueberlegungen lassen sich aber unabhängig von den notwendigen Reformen in den Entwicklungsländern einige Folgerungen für die schweizerische Entwicklungs- und Aussenwirtschaftspolitik ableiten. ${ }^{23}$ Diese entstehen einerseits aus den unterschiedlichen Wirkungen der einzelnen Transaktionsformen, andererseits aus der hohen internationalen Verflechtung der schweizerischen Wirtschaft.

(1) Nutzung des Wirtschaftspotentials:

Es macht grundsătzlich wenig Sinn, dass die Entwicklungszusammenarbeit aus einem Land wie der Schweiz sich auf die Wissensvermittlung in Bereichen konzentriert, die sich die Schweiz nur dank einem unverhältnismässigen Protektionismus erhalten konnte und somit dort also komparative Nachteile besitzt. Zwar hat sich im letzten Jahrzehnt - wie die Jahresbe-

23. Einige Ueberlegungen hierzu finden sich bereits in R. Weder (1990), Die Probleme der Schaffung eines industriellen Gewebes, in: Gern, J.-P. et al. (Hrsg.), L'industrialisation défi ou opportunité pour les pays pauvres?, Universität Neuchâtel, S. 66 77. 
richte der DEH zeigen - einiges geăndert, aber 1988 fielen immer noch 27\% der bilateralen Entwicklungszusammenarbeit der DEH auf den Sektor Land- und Forstwirtschaft und kamen $27 \%$ der vom Bund verpflichteten Experten aus der Berufsgruppe 'Landwirtschaft'. ${ }^{24}$ Es gilt in Zukunft vermehrt das Know-how in denjenigen Bereichen zu vermitteln, in denen die Schweiz führend ist und das in vielen Entwicklungslăndern für Systemreformen auch dringend benötigt wird (z.B. Aufbau von Finanz- und Kreditsystemen, Etablierung von Rechtssystemen, Errichtung von Bildungssystemen, Entwicklung von technischen Făhigkeiten im Handwerk). Warum also nicht die Entwicklungszusammenarbeit vermehrt an den eigenen Făhigkeiten orientieren und die Schweizer Firmen, die ohnehin in den Entwicklungslăndern tătig sind, vermehrt in die Entwicklungspolitik einbinden?

(2) Integration der Aussenwirtschafts- und Entwicklungspolitik:

Zwar ist die Trennung der schweizerischen Aussenpolitik in die Aussenwirtschafts- (BAWI)25 und Entwicklungspolitik (DEH) polit-ökonomisch - das heisst aufgrund der Interessenstruktur - erklärbar, sie wird aber okonomisch in einem international so vielfältig verflochtenen Land wie der Schweiz immer fragwürdiger. Eine glaubwürdige Aussenpolitik müsste vermehrt versuchen, beide wirtschaftspolitischen Teilbereiche nach denselben Grundsătzen zu handhaben. Vor allem bei einem vermehrten Einbeziehen des Know-hows der Wirtschaft ist die Trennung kaum mehr aufrechterhaltbar. Oder macht es Sinn, durch aussenwirtschaftspolitische Massnahmen den Export von Investitionsgütern zu fördem und auf der anderen, entwicklungspolitischen Seite solche Unterfangen für die Entwicklungslănder nicht zu unterstützen oder gar in Frage zu stellen?

(3) Förderung internationaler Kooperationsformen:

$\mathrm{Da}$, wie oben gezeigt, die internationalen Kooperationsformen eine Reihe von Vorteilen haben im Vergleich zum Handel von Gütem oder zur auslăndisch kontrollierten Direktinvestition, sollte in der Entwicklungszusammenarbeit vermehrt das Potential von Joint Ventures und anderen Kooperationsformen genutzt werden. Obwohl diesbezüglich zwar konkrete Wirkungsanalysen noch auf ihre Realisierung warten, könnte es z.B. interessant sein, ein Kreditsystem oder ein Patentsystem zusammen mit Partnern aus Entwicklungslăndern, dem Bund und Schweizer Banken oder Beratungsfirmen zu entwickeln und zu implementieren. Die Verbindung von wirtschaftlichem und öfentlichem Interesse ist oft nicht schädlich, sondern kann positive Wirkungen zeigen. Das BAWI unterstützt heute schon - in allerdings bescheidenem Umfang - Institutionen wie die UNIDO (Zürich) oder

24. Vgl. DEH (1989), Entwicklungszusammenarbeit der Schweizerischen Eidgenossenschaft. Jahresbericht 1989, S. 25 und 34.

25. BAWI = Bundesamt für Aussenwirtschaft. 
die TFTP (Genf), ${ }^{26}$ die primăr Kooperationspartner aus der Schweiz für Firmen aus Entwicklungslăndem suchen und vermitteln. Solche Tătigkeiten müssten und könnten in Zukunft noch vermehrt gefordert werden, mit - wie einige Erfolgsbeispiele zeigen - entsprechend grosseren Wirkungen. Diese Politik müsste auch in der DEH verankert werden.

(4) Informations- anstatt Technologietransfer:

Aufgrund der obigen Ueberlegungen ist es okonomisch nicht sinnvoll, eine ganz bestimmte Technologie - sei sie nun angepasst oder nicht - in der Schweiz zu entwickeln und in die Dritte Welt zum Nulltarif zu exportieren. Denn letztlich muss der Markt entscheiden, was angepasst ist oder nicht; zu viele sogenannte angepasste Technologien enwiesen sich plotzlich als unangepasst. Oder basiert ein Teil des internationalen Erfolges von Südkorea oder Taiwan nicht gerade auf in der entwicklungspolitischen Debatte oft als 'unangepasst' bezeichneter (Gross)Technologie? Was hingegen meines Erachtens auch aufgrund gewisser 'natürlicher Marktunvollkommenheiten' vertreten werden kann, ist die Bereitstellung des offentlichen Gutes 'Information'. Da es vielen Entwicklungslăndern an Informationen über potentielle Exportmärkte oder eben über Kooperationspartner, aber auch an Marktinformationen über mögliche Exportprodukte fehlt, wäre es der wirtschaftlichen Entwicklung dieser Länder sehr förderlich, wenn solche Informationen bereitgestellt und konkret vermittelt würden. ${ }^{27}$

Die Notwendigkeit von institutionellen Reformen in vielen Entwicklungslăndern, die hohe Bedeutung der wirtschaftlichen Entwicklung und der gleichzeitig vielfăltigen wirtschaftlichen Verflechtung der Schweiz sowie die zunehmende Fragwürdigkeit von Dualismen, wie "angepasste" und "unangepasste" Technologie oder "Technologie" und "Information", wird dazu führen, dass die schweizerische Entwicklungspolitik grundsătzlich überdacht werden muss und dass neue Schwerpunkte zu setzen sind. Dabei wird die Aussenwirtschaftspolitik nicht unberührt bleiben. Vielmehr müssen beide Teilbereiche in eine kohărente und konsistente Aussenpolitik integriert werden.

26. TFTP $=$ Technology for the People.

27. Wie ein Seminar unter der Leitung von Prof. J. Forster (IUED) in Chexbres zum Thema "Vers de nouvelles Formes de Transfert de Technologie?" zeigte, bestehen diesbezüglich interessante Ideen, die von gemeinsamen Zeitschriften bis zu Seminaren von schweizerischen technischen Hochschulen und Assistentenaustauschen reichen; nur die Finanzierung scheint ein noch nicht gelöstes Problem zu sein. 